\title{
Silicon Microspheres
}

\author{
Ali Serpengüzel* \\ Koç University, Microphotonics Research Laboratory, Physics Department, \\ Rumeli Feneri Yolu, Sariyer, Istanbul 34450 Turkey
}

\begin{abstract}
Morphology-dependent resonances are observed in silicon microspheres, both in the transmission and elastic scattering spectra in the O-band. Approximately $23 \%$ of the power is coupled out at the resonance wavelength. The highest observed quality factor for the morphology dependent resonances was on the order of $10^{5}$. These resonances have a linewidth of $0.007 \mathrm{~nm}$ and a mode spacing of $0.19 \mathrm{~nm}$.
\end{abstract}

Keywords: Channel dropping filter, dielectric microsphere, evanescent coupling, integrated optoelectronics, microsphere resonator, morphology dependent resonances, optical resonance, optical coupler, optical fiber, whispering gallery modes.

\section{INTRODUCTION}

Silicon has been the material of choice for the electronics industry for more than half-a-century. It is a relatively inexpensive, plentiful, and well understood material for producing electronic devices $\left[{ }^{1}\right]$. Additionally, the need for low cost photonic devices has stimulated a significant amount of research in silicon photonics $\left[^{2},{ }^{3}\right]$. Although silicon photonics is less well developed as compared to III-V technologies; it is poised to make a serious impact on the optical communications industry, as well as in many other photonic applications $\left[{ }^{4}\right]$. Recent progress in silicon photonics is being heralded by the observation of stimulated Raman scattering (SRS) first in pulse $\left.{ }^{5}{ }^{6},\right]$ and later in $\mathrm{CW}\left[{ }^{7}\right]$ silicon Raman lasers.

Microsphere resonators are uniquely applicable in compact optoelectronic devices in wavelength division multiplexing (WDM) applications $\left[{ }^{8}\right]$. WDM has the advantage of increasing the bandwidth of the current fiber optic networks. In WDM, the final optical to electronic conversion needs an all-optical packet-switching layer, which consists of all optical gates, interferometers, semiconductor optical amplifiers, resonant cavity enhanced (RCE) photodetectors, optical random access memory (RAM) elements, and channel dropping filters. In these planar lightwave circuits, dielectric microspheres can be used as compact channel dropping filters.

In this paper, we report the silicon microsphere based optical channel dropping. The MDR's in the elastic spectrum and associated dips in the transmission spectrum are experimentally observed by coupling of light between an optical fiber half coupler (OFHC) and the MDR's of a silicon microsphere placed on the OFHC surface. Silicon microspheres with their MDR's and Q-factor of $10^{5}$ can be used as active channel-dropping filters.

\section{MORPHOLOGY DEPENDENT RESONANCES}

The MDR's of the microsphere can be understood using geometrical optics. As the light propagates around the microsphere, it is confined by almost total internal reflection (TIR) [ $\left.{ }^{9}\right]$. After circumnavigating the microsphere, the

\footnotetext{
* Correspondence should be addressed to Ali Serpengüzel, aserpenguzel@ku.edu.tr; phone: + 90 (212) 338-1312; fax: + 90 (212) 3381547, http:/home.ku.edu.tr/ aserpenguzel, http://microphotonics.ku.edu.tr, Koç University, Microphotonics Research Laboratory, Physics Department, Rumeli Feneri Yolu, Sariyer, Istanbul 34450 Turkey.
}

Laser Beam Control and Applications, edited by Alexis V. Kudryashov, Alan H. Paxton, Vladimir S. Ilchenko, Adolf Giesen, Detlef Nickel, Steven J. Davis, Michael C. Heaven, J. Thomas Schriempf, Proc. of SPIE Vol. 6101, 61010K, (2006) · 0277-786X/06/\$15 · doi: 10.1117/12.669575 
light wave returns to the original starting point in phase to interfere constructively with itself. This constructive interference can occur only at certain discrete MDR wavelengths $\left[{ }^{10}\right]$. Each MDR is characterized by a mode number (n) and a mode order (1). Physically, (n) indicates the number of nodes in the internal intensity distribution as the polar angle is varied from $0^{\circ}$ to $180^{\circ}$. The mode order (l) indicates the number of nodes in the internal intensity distribution in the radial direction. For each set of mode number (n) and mode order (l), there is a transverse electric (TE) and transverse magnetic (TM) MDR. For a given microsphere, the MDR occurs at specific value of the size parameter, $\mathrm{x}_{\mathrm{n}, 1}$, which is given by $2 \pi \mathrm{a} / \lambda_{\mathrm{n}, 1}$, where $\lambda_{\mathrm{n}, 1}$ is the light wavelength in vacuum and a is the radius of the microsphere. These MDR's have been verified experimentally at optical wavelengths with micrometer sized spheres.

The greatest impediment to the use of microsphere resonators in practical devices has been the difficulty of efficiently light coupling into and out of the spheres. To couple light into or out of the microsphere, it is necessary to overlap the evanescent field of the MDR with the evanescent field of the TIR. Such coupling has been implemented, by the use of thin tapered optical fibers, planar waveguide couplers, side-polished optical fibers, or high-indexprisms. If a microsphere is placed near the angled surface, and within the evanescent field of the fiber-optic core, then there is an efficient energy exchange in resonance between the waveguide mode of the fiber and the MDR of the microsphere $\left[{ }^{11}\right]$.

\section{GEOMETRY AND EXPECTED RESULTS}

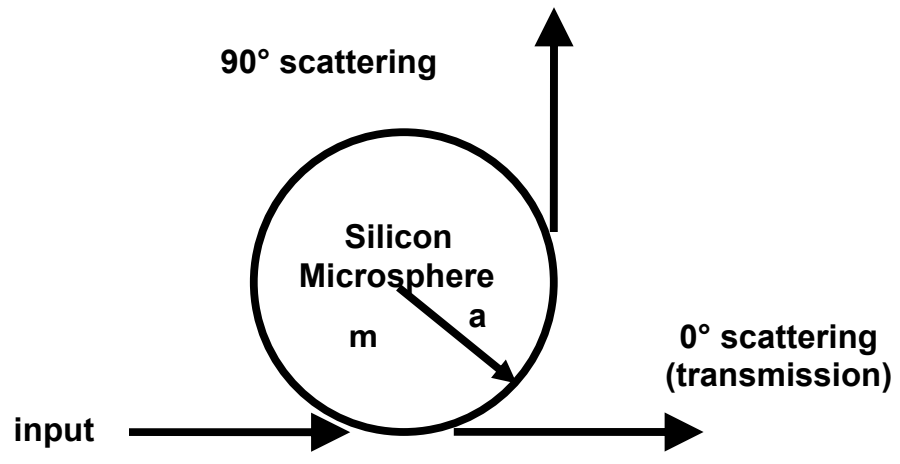

Fig.1. Elastic light scattering geometry from a silicon microsphere.

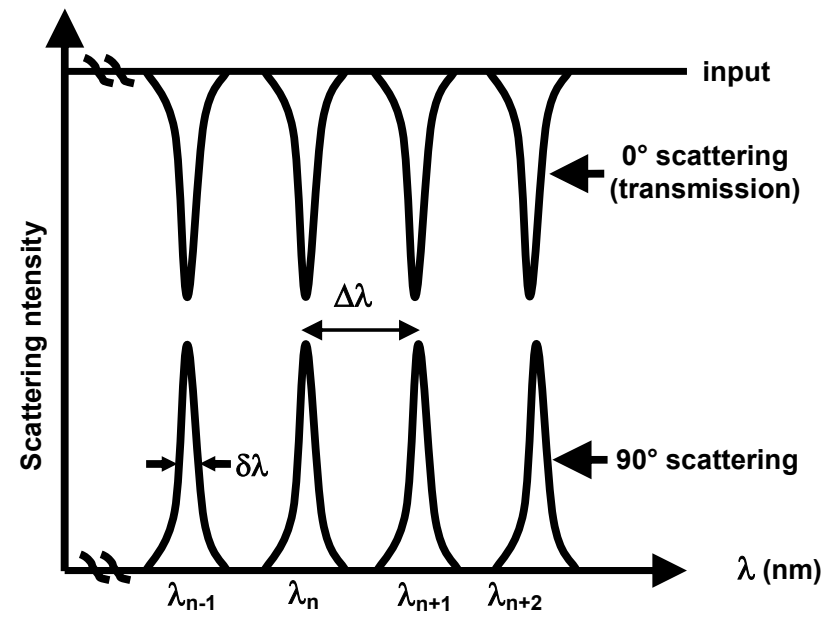

Fig.2. Estimated elastic light scattering spectra from a silicon microsphere. 
Elastic light scattering calculations are based on the geometry shown in Fig. 1. A silicon microsphere (with refractive index of 3.5) surrounded with air is placed on an optical fiber half coupler (OFHC), and the $90^{\circ}$ scattered and transmitted signals can be detected. A tunable laser can be used to excite the MDR's of the silica microsphere. The estimated spectra at scattering angles of $90^{\circ}$ and $0^{\circ}$ (forward scattering or transmission) are shown in Fig. 2.

\section{OPTICAL CHANNEL DROPPING}

In the experiments, a tunable distributed feedback (DFB) laser is used to excite the MDR's of the silicon microsphere. Wavelength tuning is achieved by tuning the temperature of the DFB laser with a laser diode controller (LDC). Laser light is coupled into the OFHC. The optical fiber used in the OFHC is a standard $1300 \mathrm{~nm}$ single-mode fiber. The silicon $\mu$ sphere used in the experiment with $a=530 \mu \mathrm{m}$ and $n=3.5$ is placed on the interaction region of the OFHC. The scattered light from the microsphere at $90^{\circ}$ is collected by a microscope lens is measured by an InGaAs photodiode. The transmitted power through the optical fiber is measured by an optical multimeter (OMM) with InGaAs power/wave head (PWH). The InGaAs photodiode signal is sent to the digital oscilloscope for signal monitoring and data acquisition. An infrared viewer is used to observe scattered light from the $\mu$-sphere. Data acquisition and control are performed with IEEE-488 GPIB interface.

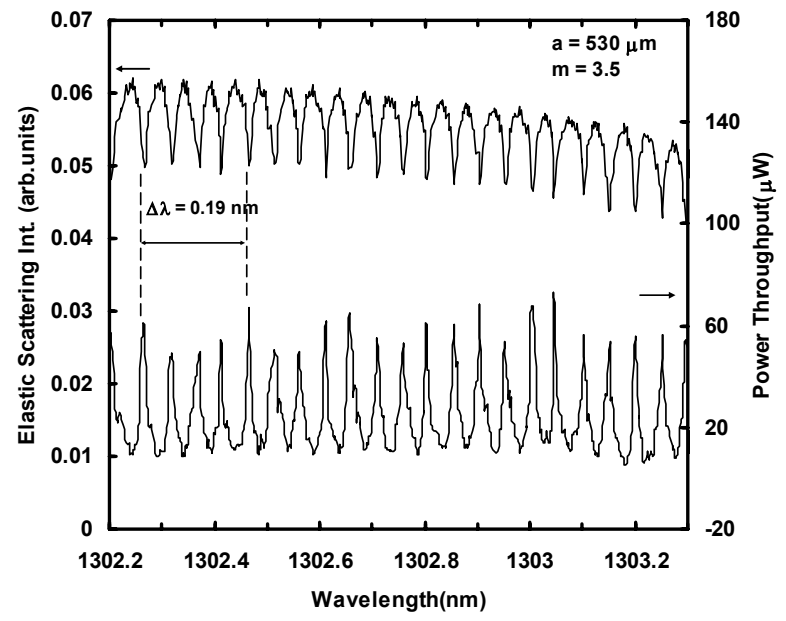

Fig. 3. Elastic scattering spectrum and power transmission spectrum from the silicon microsphere.

Fig. 3 shows the elastic scattering spectrum at a scattering angle of $90^{\circ}$ from the silicon microsphere and the power transmission spectra at the end of the optical fiber. There is a background due to OFHC surface imperfections. The temperature of the DFB laser is tuned from $18^{\circ} \mathrm{C}$ to $34^{\circ} \mathrm{C}$ at a constant current of $29 \mathrm{~mA}$. The temperature range corresponds to $1302.18 \mathrm{~nm}$ to $1303.30 \mathrm{~nm}$. The dips in the transmitted signals are due to the coupling to the MDR's of the microsphere.

\section{CONCLUSIONS}

We have demonstrated the excitation of MDR's of silicon and glass microspheres using an optical fiber half coupler (OFHC) and, a temperature tunable distributed feedback (DFB) laser. MDR peaks in the elastic scattering spectra and associated dips in the transmission spectra are observed experimentally. With the proper system design, it would be possible to totally drop the selected MDR's power from the transmission spectrum. The microsphere, optical fiber, and detector system shows promise as a building block for optoelectronic integration.

\section{ACKNOWLEDGMENTS}

We would like to acknowledge the partial support of this research by the European Commission Grant No: FP6-IST511616: PHOREMOST and FP6-IST-003887 NEMO.

\section{REFERENCES}


[1] L. Pavesi, "Will silicon be the photonic material of the next millennium?," J.Phys.: Condensed Matter, vol. 15, pp. 11691196, 2003.

[2] L. Pavesi and D. J. Lockwood, Silicon Photonics. New York: Springer, 2004.

[3] G.T. Reed and A.P. Knights, Silicon Photonics: An Introduction. John Wiley, 2004.

[4] O. Boyraz and B. Jalali, "Demonstration of a silicon Raman laser," Opt. Exp., vol. 12, pp. 5269-5273, Oct. 2004.

[5] H. Rong et. al., "An all -silicon Raman laser," Nature, vol. 433, pp. 292-294, Jan. 2005.

[6] O. Boyraz and B. Jalali, "Demonstration of directly modulated silicon Raman laser," Opt. Exp., vol. 13, pp. 796-800, Feb. 2005.

[7] H. Rong et al., “A continuous-wave Raman silicon laser,” Nature, vol. 433, pp. 725-728, Feb.2005.

[8] A. Serpengüzel, S. Arnold, G. Griffel, and J. A. Lock, "Enhanced coupling to microsphere resonances with optical fibers,” J. Opt. Soc. Am. B., vol. 14, no. 4, pp. 790-795, Apr. 1997.

[9] M. Pelton and Y. Yamamoto, "Ultralow threshold laser using a single quantum dot and a microsphere cavity", Phys. Rev. A, vol. 59, no. 3, pp. 2418-2421, March 1999.

[10] B. R. Johnson, "Morphology-dependent resonances of a dielectric sphere on a conducting plane", J. Opt. Soc. Am. A, vol. 11, no. 7, pp. 2055-2064, July 1994.

[11] J.-P. Laine, B. E. Little, and H. A. Haus, "Etch-Eroded Fiber coupler for whispering-gallery-mode excitation in high Q silica microspheres", IEEE Photon. Technol. Lett., vol. 11, no. 11, pp. 1429-1430, Nov. 1999. 